\title{
Clinical and Electroencephalographic Findings in Patients with Sepsis-associated Encephalopathy and the Evaluation of Their Effects on Survival
}

\author{
(D) Günseli Orhun ${ }^{1}$, (D) Nerses Bebek2 , (D) Perihan Ergin Özcan ${ }^{1}$, (D) Achmet Ali1 , (D) Figen Esen ${ }^{1}$ \\ 1istanbul University, Istanbul Faculty of Medicine, Department of Anesthesiology and Intensive Care, Istanbul, Turkey \\ 2 istanbul University, istanbul Faculty of Medicine, Department of Neurology, Istanbul, Turkey
}

\section{Abstract}

Objective: Acute brain dysfunction is common in sepsis patients and is associated with increased mortality. This study aimed to investigate the clinical and electroencephalographic (EEG) findings of patients with acute neurological dysfunction due to sepsis and to evaluate their relationship with prognosis.

Methods: Sixty-one patients with sepsis were included in this prospective observational study. All patients underwent a daily neurological examination. EEG monitoring was performed in patients with delirium, coma, clinical seizures, and focal neurological findings. The patients were divided into two groups as survivors and non-survivors.

Results: Sixty-one patients included in the study had a median age of 60 years (range=21 to 81), Acute Physiology and Chronic Health Evaluation 2 score of 24 (range=14 to 39), and Sepsis-related Organ Failure Assessment score of 10 (range=3 to 24). During the EEG examination, coma was detected in 23 patients (38\%), delirium/confusion in 20 patients (33\%), clinical seizures in 16 patients (26\%) and focal neurological findings in two patients (3\%). The overall mortality rate was $42 \%(n=26)$. The EEG examination showed slowing of background activity in all patients. Also, among three patients who had seizure activity, two showed findings of an electrophysiological seizure. The EEG examination revealed a relationship between low-amplitude non-reactive background activity and mortality.

Conclusion: EEG monitoring may be indicated in the follow-up of encephalopathy in sepsis, the determination of the severity of the disease, the presence of an accompanying seizure or status, and prognosis.

Keywords: Sepsis, brain dysfunction, electroencephalography, prognosis

\section{INTRODUCTION}

Acute brain dysfunction is a severe complication of sepsis and often occurs before alterations in other organ functions in the early period $(1,2)$. Clinically, it manifests itself in the form of acute neurological changes, such as altered consciousness, which may vary from delirium to coma, or a seizure activity (3). The relationship between these neurological findings in sepsis and increased mortality has been shown in several studies. The complication draws attention as an essential risk factor for the development of long-term cognitive impairment in survivors $(4,5)$.
The pathophysiology of brain dysfunction in sepsis is multifactorial. In these patients, where infection does not directly affect the central nervous system (CNS), brain dysfunction is associated with neuroinflammatory and ischemic processes that result in impaired neuronal function $(3,6)$. Also, the clinical picture is aggravated by the dysfunction of other organs, electrolyte disturbances, and the use of potential neurotoxic drugs such as antibiotics. Electroencephalography (EEG) changes observed in sepsis patients may reflect these neurotoxic processes (7-9). Although EEG in sepsis is a monitoring method used to diagnose 
brain dysfunction and to determine its severity and prognosis, the literature does not have sufficient data on this subject (10). During sepsis, various EEG findings are observed, such as the absence of reactivity, slowing of the background activity (the presence of theta and delta activity), electrophysiological seizures and periodic discharges $(8,11,12)$. The frequency of these changes varies according to the design of the study (prospective or retrospective), the studied patient population [surgical or medical intensive care unit (ICU)], the severity and duration of sepsis (acute phase or late period), and the recording method of EEG (standard EEG recording or continuous EEG recording). Although some abnormalities, such as a triphasic wave pattern, a delta frequency, or a burst-suppression pattern, seen in the EEG examinations of sepsis patients are thought to be associated with poor prognosis, these findings need to be supported by prospective studies (10). Also, since non-convulsive seizures and status epilepticus are common in sepsis patients, the presence of ictal EEG findings may be guiding in diagnosis and treatment as well as be a potential prognostic marker $(10,13)$.

This study aimed to investigate the clinical and EEG findings of patients who were hospitalized in the ICU due to sepsis and who had acute changes in their neurological status, and to evaluate their relationship with prognosis.

\section{METHODS}

\section{Patients and Protocol}

This prospective observational study was performed between February 2013 and February 2017 at the Medical and Surgical ICU of the istanbul Faculty of Medicine at İstanbul University. Patients over 18 years of age, who were diagnosed with severe sepsis and septic shock according to the international diagnostic criteria for sepsis (14) and who were hospitalized in the ICU for more than 48 hours and who showed clinical signs of acute brain dysfunction, were included in the study. Acute brain dysfunction was defined as delirium/confusion, coma, clinical seizure, and the presence of focal neurological findings in neurological examination (15). Patients with a known CNS disease (neurodegenerative, inflammatory, cerebrovascular, neoplastic, traumatic brain injury) or CNS infection before the critical disease were excluded from the study. Also, patients with severe dementia, acute or chronic hypoxic brain injury affecting the neurological assessment, and patients with the presence of clinical evidence of brain death were excluded from the study. Patients who had severe hepatic insufficiency, who continuously needed muscle relaxants or who had a body temperature of $<35$ ${ }^{\circ} \mathrm{C}$ were also excluded from the study. The study was approved by the Institutional Review Board (approval number: 2013-98), and written consent was obtained from patients or their close relatives for creating medical records and applying the study protocol.

After the patients were included in the study, their clinical and demographic data, including age, gender, co-morbidities, the reason for admission to the ICU and admission category (medical or surgical), history of neurological disease, days in the ICU and duration of hospitalization were recorded. Acute Physiology and Chronic Health Evaluation (APACHE 2) score and the Simplified Acute Physiology score (SAPS 2) were used to assess the disease severity at enrollment. The Sepsis-related Organ Failure Assessment (SOFA) score was used to assess the severity of organ dysfunction from the time of admission to the ICU to discharge, and the evaluations were made daily. During the EEG monitoring, the renal and hepatic SOFA scores were calculated to evaluate the renal and hepatic failure, respectively.

From the time of admission to the ICU and throughout the study, vital and clinical examination findings of the patients were recorded. Patients were regularly followed-up with a standard laboratory protocol, including complete blood count, blood biochemistry, and blood gas analysis. From admission to the day of discharge, vital parameters, use of continuous sedation and the type of administered drug, days of sedation, duration of septic shock, days of mechanical ventilation, and days of hemofiltration were recorded daily. The infection focus that caused sepsis was noted along with culture results. Sepsisinduced hypotension was defined as a systolic blood pressure (SBP) of $<90 \mathrm{mmHg}$ or a mean arterial pressure of $<70 \mathrm{mmHg}$, or as a decrease in SBP $>40 \mathrm{mmHg}$ or, in the absence of other causes, a decrease of beyond two standard deviations below the normal range for that age. Septic shock was defined as persistent sepsis-induced hypotension despite adequate fluid resuscitation in the absence of other causes of hypotension (14).

\section{Neurological Examination}

After the patients were admitted to the ICU, a detailed neurological examination was performed. All patients were followed up with a sedation protocol, except for the daily sedation discontinuation periods in the ICU. In patients receiving sedation, the depth of sedation was evaluated with the Richmond Agitation-Sedation scale (RASS) after the daily sedation was discontinued (16). The delirium/confusion assessment of the patients responding to the verbal stimulus during the RASS examination was made using the confusion assessment method for the ICU (CAM-ICU) (17), and the assessment was performed twice daily by a trained ICU nurse or 
an ICU specialist. Patients who responded to the verbal stimulus and had a RASS score of $\geq-3$ (for example, a RASS score between -3 and +4 ) were diagnosed with delirium when at least one test was positive on the CAM-ICU test. Patients with a RASS score of -4 (responding only to physical stimulus) and -5 (completely unresponsive) were considered to be in a coma, and their level of consciousness was assessed using the Glasgow Coma scale (GCS). Coma was defined as GCS $\leq 8$ in non-sedated patients or after three days of discontinuation of sedation in previously sedated patients (18). The presence of generalized or focal, tonic or clonic, short or long term and recurrent movement on the face or extremities was defined as an epileptic seizure. Any lateralized deficit was considered a focal neurological deficit.

\section{EEG Monitoring and Analysis}

Following the discontinuation of the sedation protocol as described above for the neurological evaluation of the patients that received sedation, sedation was discontinued at the time of EEG recording in patients with appropriate clinical and neurological status in ICU follow-up and treatment period. During the EEG recording, continuous sedative drug infusion was performed following internationally accepted standards in patients who required sedation, and the type of sedative drug used was recorded. The EEG monitoring of all patients with acute brain dysfunction as a result of the neurological examination was performed by an EEG technician using a portable EEG device (MICROMED SAM 32 RFO C1, 2008) at the bedside in the ICU. Twenty-one electrodes placed according to the international 10-20 system were used in scalp recording. EEG recording was performed for a median of 51 (range $=20-1482)$ minutes. The analysis of the EEG recordings was performed by a neurologist (NB) who was a specialist in clinical neurophysiology. The American Clinical Neurophysiology Society's standardized "Critical Care EEG Terminology" guidelines were used in evaluating the EEG (19). The Salzburg consensus criteria were used in evaluating the non-convulsive seizures (20).

\section{Statistical Analysis}

Basic demographic and clinical data were presented as median and minimum-maximum for the continuous variables, and frequency and percentage for the categorical variables. The patient group was divided into two groups as survivors and nonsurvivors. In intergroup comparisons, the chi-square test was used for the categorical variables and the Mann-Whitney $U$ test for the quantitative variables. A $p$ value of $<0.05$ was considered statistically significant. All statistical analyses were performed using SPSS for Windows 15.0 software (SPSS Inc., Chicago, IL, USA).

\section{RESULTS}

\section{Demographic and Clinical Data of the Study Patients}

Between February 2013 and February 2017, 448 patients were admitted to our ICU with the diagnosis of sepsis. Three hundred and eight patients with acute brain dysfunction were evaluated for the study, and as a result, 61 sepsis patients (30 females, 31 males) who underwent EEG monitoring were included in the study. The flowchart of the study is shown in Figure 1. The median age of the patients was 60 (range $=21-87$ ) years, 51\% $(n=31)$ of the patients were admitted to the ICU for medical reasons, and $49 \%(n=30)$ for surgical reasons. During the ICU follow-up, 52 patients (84\%) developed septic shock, 27 patients (44\%) underwent hemofiltration, and all patients underwent mechanical ventilation. Baseline median APACHE 2, SOFA and SAPS 2 scores were 24 (range $=14-39), 10$ (range $=3-24$ ) and 53 (range $=15-90)$, respectively. Twenty-six patients (42\%) died during the ICU follow-up (Table 1).

\section{Clinical and Neurological Findings of the Patients during EEG Monitoring}

A total of 83 EEG recordings were performed in 61 patients included in the study (14 patients underwent a second EEG, seven patients a third, one patient a fourth one). The median EEG recording time was 51 (range $=20-1482)$ minutes. The median time until the development of acute brain dysfunction was 6 (range=1-30) days after admission to the ICU. During the EEG monitoring, the most common neurological finding was coma (38\%), followed by delirium/confusion (33\%), clinical seizure (26\%), and focal neurological findings (3\%). The EEG examinations revealed that $90 \%$ of the patients had a significant slowing in background activity, with the most common wave frequency being the combination of theta/delta $(n=24,41 \%)$ followed by theta $(n=24,39 \%)$, and delta slow waves $(n=6,10 \%)$. Two patients with an EEG wave frequency of theta/delta and one patient with a delta frequency were accompanied by frontal intermittent rhythmic delta activity. No reactivation was observed in EEG in 47 patients (77\%). In six patients (10\%), background activity was observed to consist of low amplitude non-reactive rhythms. Generalized periodic epileptiform discharge was observed in one patient, periodic lateralized epileptiform discharge (PLED) in one, and a triphasic wave pattern in four patients. During the EEG examination in three patients, the presence of seizure activity, two of them being electrophysiological, was observed (Table 2). It was noteworthy that one of these patients was accompanied by PLEDs for a few seconds at the beginning of the electrophysiological seizure activity. The other patient had a recurrent twitching in the half of the face, which resolved after 
intravenous injection of midazolam. Considering this finding as a focal motor seizure, levetiracetam treatment was begun, and the seizures disappeared in the following period. The clinical, neurological, and prognosis findings of the patients with specific EEG findings are presented in Table 3, and the EEG recording samples are presented in Figures 2-4. The follow-up EEG examination revealed that EEG findings had improved in five patients, remained the same in four patients, and worsened in five patients. During the EEG monitoring, the median SOFA score, which indicated the severity of organ dysfunction, was 9 (2-20), the median renal SOFA was 2 (0-4), and the median hepatic SOFA was $0(0-4)$. During the EEG monitoring, $41 \%$ of the patients $(n=25)$ received continuous sedative infusion, and $36 \%$ of the patients $(n=22)$ were given remifentanil, $7 \%(n=4)$ dexmedetomidine, and $2 \%(n=1)$ midazolam for sedation. When the EEG findings of the patients who were given sedation during EEG recording were compared to those who were not given sedation, no difference was found in terms of background activity ( $p=0.575)$, amplitude $(p=0.669)$, and the absence of reactivation $(p=0.871)$.

\section{Evaluating the Relationship Between Clinical Features and EEG Findings and Clinical Outcomes}

When the survivors $(n=35)$ and non-survivors $(n=26)$ were compared, the age of the non-survivors was higher than the survivors ( $p=0.014)$, and the distribution of genders was similar $(p=0.051)$. The SOFA score $(p=0.017)$ was higher at admission to the ICU, and no significant difference was found between other the disease severity scores. The total SOFA and renal SOFA scores of non-survivors during the EEG monitoring were significantly higher $(p=0.001$ and $p=0.005$, respectively); there was no difference between the groups in terms of hepatic SOFA score $(p=0.084)$. The survivors had a higher prevalence of delirium/confusion $(p=0.013$ ), while coma was a more common neurologic finding in the non-survivors ( $p=0.025)$. There was no significant difference in terms of clinical seizure rates $(p=0.915)$. The non-reactive EEG pattern consisting of low amplitude nonreactive rhythms was more prevalent in the EEG examination of the non-survivors ( $p=0.034)$; however, there was no difference between the groups in terms of background activity $(p=0.192)$, low amplitude $(p=0.108)$, and the absence of reactivation $(p=0.984)$ (Table 4).

\section{DISCUSSION}

In this prospective observational cohort study using EEG in sepsis patients with acute brain dysfunction, we found that: 1) all patients had abnormal EEG findings, primarily the slowing of background activity; 2) the presence of low-amplitude nonreactive rhythms on EEG were correlated with mortality; 3) advanced age, the SOFA score at admission and a high SOFA score at the time of EEG were correlated with mortality.

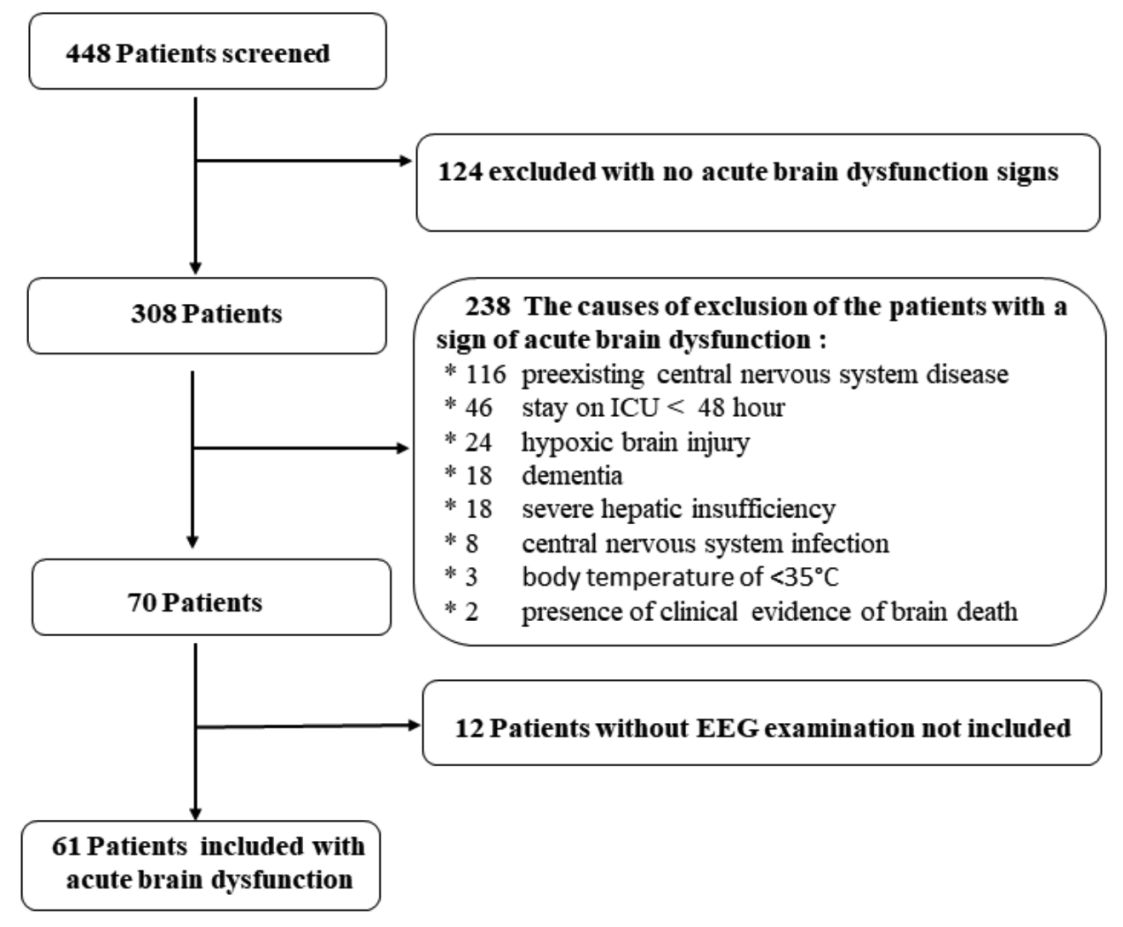

Figure 1. Flow chart of inclusion and exclusion of patients EEG: Electroencephalography, ICU: Intensive care unit 
It is known that acute neurological dysfunction developing in ICU patients with sepsis is associated with morbidity and mortality. Acute brain dysfunction in sepsis may involve different pathophysiological pathways $(3,6)$. Whatever the underlying mechanism, various examination methods are used in addition

\begin{tabular}{|c|c|}
\hline Variables & $n=61$ \\
\hline Age (years) & $60(21-87)$ \\
\hline Female, n (\%) & $30(49)$ \\
\hline APACHE 2 at admission & $24(14-39)$ \\
\hline SAPS 2 at admission & $53(15-90)$ \\
\hline SOFA at admission (from 0 to 24 ) & $10(3-24)$ \\
\hline Maximum SOFA (from 0 to 24 ) & $12(4-24)$ \\
\hline Glasgow Coma score at admission & $15(4-15)$ \\
\hline $\begin{array}{l}\text { Admission category, } \mathrm{n}(\%) \\
\text { Medical } \\
\text { Surgery }\end{array}$ & $\begin{array}{l}31(51) \\
30(49)\end{array}$ \\
\hline $\begin{array}{l}\text { Systemic diseases, n (\%) } \\
\text { Cardiac disease } \\
\text { Diabetes mellitus } \\
\text { Chronic renal failure } \\
\text { Cancer } \\
\text { Respiratory disease }\end{array}$ & $\begin{array}{l}29(48) \\
22(36) \\
14(23) \\
20(33) \\
4(7)\end{array}$ \\
\hline $\begin{array}{l}\text { Site of infection, n (\%) } \\
\text { Pneumonia } \\
\text { Intraabdominal } \\
\text { Soft tissue infection } \\
\text { Urinary tract infection }\end{array}$ & $\begin{array}{l}38(62) \\
12(20) \\
5(8) \\
4(7)\end{array}$ \\
\hline $\begin{array}{l}\text { Pathogen, n (\%) } \\
\text { Pure gram negative } \\
\text { Pure gram positive } \\
\text { Polymicrobial }\end{array}$ & $\begin{array}{l}41(67) \\
3(5) \\
7(12)\end{array}$ \\
\hline Positive blood culture, n (\%) & $17(28)$ \\
\hline $\begin{array}{l}\text { Septic shock } \\
\text { Prevalence, n (\%) } \\
\text { Duration (days) }\end{array}$ & $\begin{array}{l}52(84) \\
7.5(1-36)\end{array}$ \\
\hline Duration of mechanical ventilation (days) & $15(1-41)$ \\
\hline Duration of sedation (days) & $11(1-36)$ \\
\hline $\begin{array}{l}\text { Hemofiltration } \\
\text { Prevalence, } \mathrm{n}(\%) \\
\text { Duration (days) }\end{array}$ & $\begin{array}{l}27(44) \\
6.5(1-36)\end{array}$ \\
\hline Mortality, n (\%) & $26(42)$ \\
\hline Days in the intensive care unit & $19(3-103)$ \\
\hline Duration of hospitalization (days) & $41(6-120)$ \\
\hline \multicolumn{2}{|c|}{$\begin{array}{l}\text { APACHE 2: Acute Physiology and Chronic Health Evaluation score, SOFA: Sepsis- } \\
\text { related Organ Failure Assessment score, SAPS 2: Simplified Acute Physiology score, } \\
\text { n: The number of patients (values are given as median and minimum-maximum, } \\
\text { and the qualitative data are given as frequency and percentage) }\end{array}$} \\
\hline
\end{tabular}

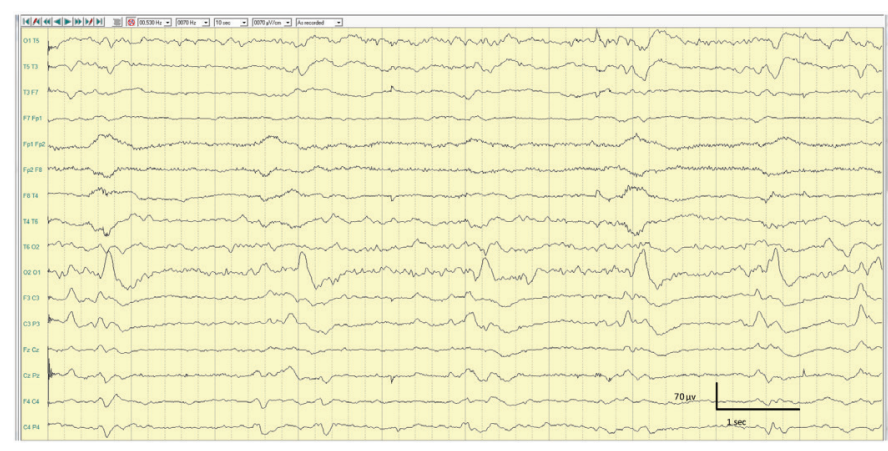

Figure 2. A representative example of EEG showing a periodic lateralized epileptiform discharge in a sepsis patient. Distinct and acute wave discharges, periodically repeating every $1.5-2$ seconds, were detected in the occipital region in the posterior half of the left hemisphere during the EEG examination of a sepsis patient who had coma on the sixth day of admission to the intensive care unit. (Circular montage. Sensitivity: 70 microvolts/cm, low frequency filter: $0.5 \mathrm{~Hz}$, high frequency filter: 70 $\mathrm{Hz}$, paper speed: 10 seconds)

EEG: Electroencephalography

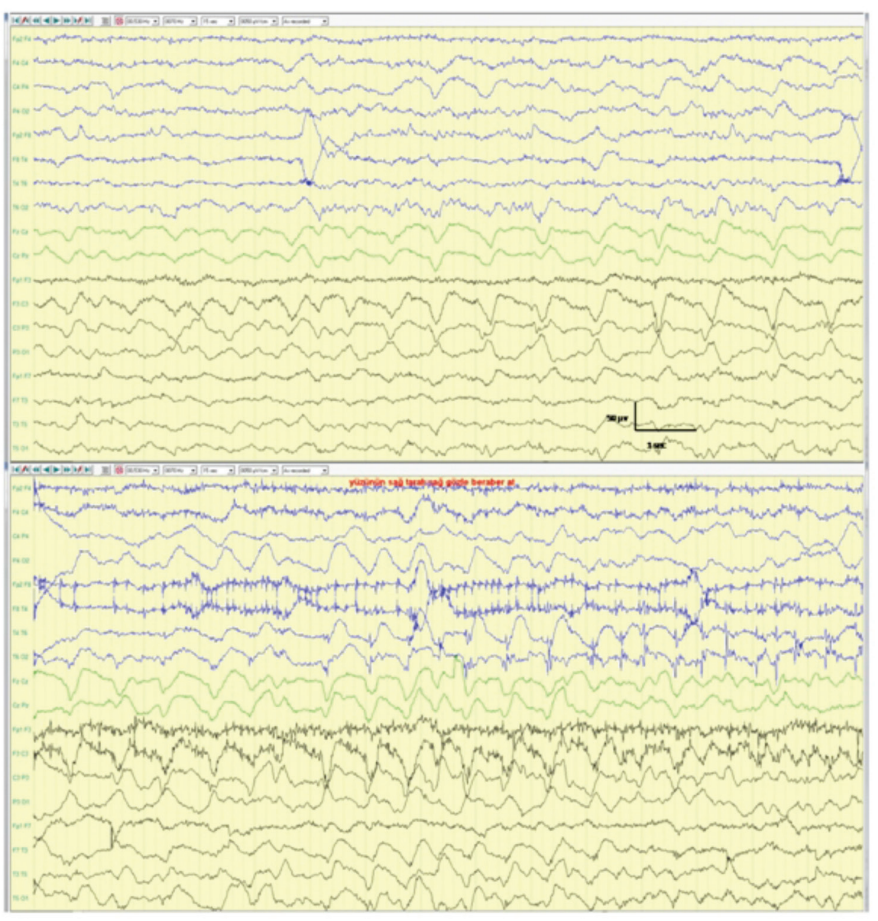

Figure 3. A representative example of EEG showing the onset of an electrographic seizure in a sepsis patient. Seizure activity in the left centro-parietal region manifested with a rhythmic, acute, slow-wave activity at $1 \mathrm{~Hz}$ of frequency, which showed evolution and was spread overall the hemisphere and the opposite hemisphere, was detected during the EEG examination of a sepsis patient who had a clinical seizure on the first day of admission to the intensive care unit. Clinically, the right side of the face was accompanied by recurrent twitching involving the eye. (Bipolar-double banana montage. Sensitivity: 70 microvolts/ $\mathrm{cm}$, low frequency filter: $0.5 \mathrm{~Hz}$, high frequency filter: $70 \mathrm{~Hz}$, paper speed: 15 seconds)

EEG: Electroencephalography 
to clinical neurological examination in order to diagnose brain dysfunction and evaluate itsseverity (21). EEG is a neuromonitoring method that provides information about the electrical activity of the brain and can be performed at the bedside. EEG provides the possibility of evaluating the causes of brain dysfunction either due to structural diseases such as traumatic brain injury, intracranial hemorrhage, cerebral ischemia, and encephalitis or to functional disorders such as metabolic and sepsis-associated encephalopathy (22). The test can be used to take early potential therapeutic measures in patients with a tendency to develop brain dysfunction, to investigate the unexplained changes in consciousness and to determine the prognosis of developing neurological complications $(13,23)$. EEG is the only examination method that can detect non-convulsive epileptic seizures (or subclinical) in critically ill patients (22). EEG is also used in the ICU to monitor the effect of sedative drugs during status epilepticus treatment $(13,22,23)$.

EEG abnormalities in sepsis patients have been evaluated in a few studies, and the number of prospective studies in the literature is limited. The type, frequency, and the prognostic value of the EEG abnormalities associated with sepsis vary in the current studies. EEG findings of sepsis-associated encephalopathy are characterized by a generalized slowing in the background activity of EEG, and the presence of theta and delta waves, which are the indicators of diffuse cortical dysfunction (24). Concerning the severity of encephalopathy, theta waves often appear in patients with mild and moderate encephalopathy (confusion, delirium), while delta activity appears in a coma, where the impairment in consciousness level is more severe. The presence of triphasic waves and a burst-suppression pattern indicates the dysfunction of deeper

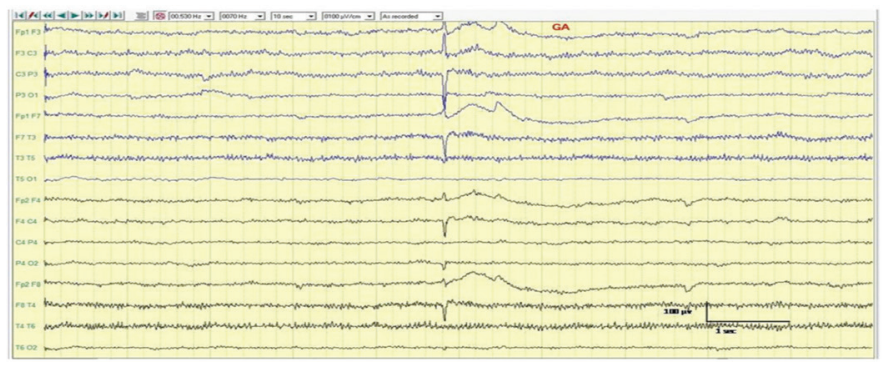

Figure 4. A representative example of EEG showing a low amplitude non-reactive rhythm in a sepsis patient. A monotone, low amplitude background activity mixed with artifacts was observed over both hemispheres during the EEG examination of a sepsis patient who had a clinical seizure on the first day of admission to the intensive care unit. It was notable that the patient's alertness did not change with noise and painful stimuli. (Bipolar-double banana montage. Sensitivity: 100 microvolts/cm, low frequency filter: $0.5 \mathrm{~Hz}$, high frequency filter: $70 \mathrm{~Hz}$, paper speed: 10 seconds)

EEG: Electroencephalography brain structures such as basal ganglia and diencephalon (10). Prospective studies on sepsis patients have reported that abnormalities in the background activity of EEG are very often $(7,11,25)$. In a prospective case series evaluating 62 patients with sepsis, $87 \%$ of the patients had abnormal EEG findings, including continuous theta and delta waves or a burst-suppression pattern, and these EEG findings have been shown to have a relationship with the diagnosis and severity of encephalopathy, as well as other organ dysfunctions and increased mortality (7). Similarly, a high prevalence in background activity abnormalities was observed in another study, but no relationship was detected between these EEG findings and the presence of sepsis-associated encephalopathy (25). A recent study in patients with sepsis demonstrated the relationship of predominant delta activity with the presence of delirium and increased mortality (11). Studies conducted on general ICU patients with and without sepsis showed that some EEG abnormalities, such as the presence of triphasic waves, delta waves, or a burst-suppression pattern, were associated with poor prognosis $(11,26,27)$. In our study, during the EEG monitoring performed upon the detection of acute brain dysfunction, we observed a slowing in the background activity in all patients, related to the presence of sepsis-associated encephalopathy. However, a significant relationship could not be established between these changes observed in the background activity and the clinical outcome. This is probably due to the small number of patients in our study.

In our study, we evaluated the EEG reactivity after giving standard noise and painful stimuli (23). The presence of reactivation in EEG requires the functional integrity of peripheral sensory pathways, the brainstem, subcortical structures, and the cerebral cortex. The absence of reactivation in EEG may be caused by the dysfunction that has developed in any of these structures, especially in the brainstem (28). The absence of reactivation in EEG with external stimulation is a sign of severe encephalopathy, and two recent studies on ICU patients with sepsis have shown its association with an increase in mortality $(11,12)$. In our study, reactivation was absent in $77 \%(n=47)$, and although a higher rate was found than the two studies mentioned above, no relationship with mortality could be established. A possible explanation for this situation is the presence of sedation, or the ongoing effects of sedative drugs, which are shown to be one of the most critical factors affecting the evaluation of reactivation during EEG recording $(11,12)$. However, in our study, there was no significant difference in terms of amplitude, slow-wave frequency, and reactivation between sedated and non-sedated patients. 
It is expected to observe the specific EEG findings less often in sepsis cases in which the CNS is indirectly affected by diseases in comparison to the cases in which the CNS is directly affected. Although it is observed less frequently, these patients have different degrees of encephalopathy, and in patients with clinical improvement, recovery from encephalopathy should be confirmed with EEG. In our study, seizures were recorded in three patients in the EEG examination. One of these patients had a clinical seizure. One case had no signs of a clinical seizure, whereas the recurrent twitching on the face of the other case was thought to be a seizure, and upon EEG examination, the presence of an electroclinical seizure was confirmed. The seizures were brought under control with treatment. Also, the presence of PLED in a patient was noted as a specific EEG finding. Furthermore, it is noteworthy that patients with a nonreactive EEG, which presents with a low amplitude and lack of reactivation, may have a worse prognosis.

It is known that the EEG findings of ICU patients are affected by sedative drugs $(10,11,13)$. Sedation is given to sepsis patients with a high disease severity score in order to ensure their safety and comfort during the treatment process. A decrease in the clearance of sedative drugs is observed during sepsis with the accompanying dysfunction

Table 2. Electroencephalography findings and simultaneous clinical variables of the patients

\begin{tabular}{|c|c|c|c|c|c|c|}
\hline & & \multicolumn{4}{|c|}{ Neurological findings during EEG monitorization } & \multirow[t]{2}{*}{ Death } \\
\hline & & $\begin{array}{l}\text { Delirium/ } \\
\text { Confusion }\end{array}$ & Coma & $\begin{array}{l}\text { Clinical } \\
\text { seizure }\end{array}$ & $\begin{array}{l}\text { Focal neurological } \\
\text { findings }\end{array}$ & \\
\hline EEG findings & n (\%) & & & & & \\
\hline \multicolumn{7}{|l|}{ Background activity-n (\%) } \\
\hline Theta & $24(39)$ & 9 & 6 & 8 & 1 & 10 \\
\hline Theta/delta* & $25(41)$ & 10 & 11 & 3 & 1 & 9 \\
\hline Delta** & $6(10)$ & - & 3 & 3 & - & 2 \\
\hline Low amplitude non-reactive background activity & $6(10)$ & 1 & 3 & 2 & - & 5 \\
\hline Low amplitude, $\mathrm{n}(\%)$ & $48(79)$ & 15 & 18 & 13 & 2 & 23 \\
\hline Absence of reactivity, $\mathrm{n}(\%)$ & $47(77)$ & 17 & 19 & 9 & 2 & 20 \\
\hline Triphasic waves, n (\%) & $4(7)$ & 2 & 1 & 1 & - & 1 \\
\hline Periodic anomalies, n (\%) & $2(3)$ & & & & & \\
\hline Generalized periodic epileptiform discharge & 1 & - & 1 & - & - & 1 \\
\hline Periodic lateralized epileptiform discharge & 1 & - & 1 & - & - & 1 \\
\hline Presence of seizures during EEG examination, $\mathrm{n}(\%)$ & $3(5)$ & - & 1 & 1 & - & 2 \\
\hline Electrophysiological seizure & $2(3)$ & & & & & \\
\hline Variables during EEG recording & $n=61$ & & & & & \\
\hline Delay from admission to neurologic signs (days) & $6(1-30)$ & & & & & \\
\hline \multicolumn{7}{|l|}{ Neurological status during EEG recording, n (\%) } \\
\hline Delirium/confusion & $20(33)$ & & & & & \\
\hline Coma & $23(38)$ & & & & & \\
\hline Presence of clinical seizure & $16(26)$ & & & & & \\
\hline Focal neurological findings & $2(3)$ & & & & & \\
\hline Duration of EEG recording, minutes & $51(20-1482)$ & & & & & \\
\hline SOFA (from 0 to 24) & $9(2-20)$ & & & & & \\
\hline Renal SOFA (from 0 to 4) & $2(0-4)$ & & & & & \\
\hline Hepatic SOFA (from 0 to 4) & $0(0-4)$ & & & & & \\
\hline Presence of sedation, $\mathrm{n}(\%)$ & $25(41)$ & & & & & \\
\hline \multicolumn{7}{|l|}{ Type of sedation, $\mathrm{n}(\%)$} \\
\hline Remifentanil & $22(88)$ & & & & & \\
\hline Dexmedetomidine & $4(16)$ & & & & & \\
\hline Midazolam & $1(4)$ & & & & & \\
\hline
\end{tabular}


of the organs such as liver or kidney, which play an essential role in the elimination of drugs. However, studies conducted on the critically ill patient group showed that EEG recordings continued to be informative about encephalopathy despite sedation (10). In our study, we applied the sedation protocol in line with international recommendations. During the EEG recording, we discontinued sedation in patients with an appropriate clinical and neurological status, and in a significant portion of patients that required sedation, we used remifentanil, which is rapidly metabolized in the plasma and the tissues. However, the effects of sedation cannot be completely ignored, even despite its discontinuation during EEG recording. In order to more clearly evaluate this condition, which is a limitation of our study, the sedative level should be measured, the neurophysiological changes during the EEG recording should be monitored, and the dose of the sedative should be determined using more advanced clinical parameters, such as the RASS score, during the EEG recording.

One of the limitations of our study was the lack of continuous EEG monitoring in all patients. An EEG with a short duration (less than 1 hour) makes the detection of the concomitant electrophysiological seizures particularly difficult. A continuous EEG provides more reliable detection of the changes in EEG,

\begin{tabular}{|c|c|c|c|c|c|c|c|c|c|c|}
\hline Case & $\begin{array}{l}\text { Age } \\
\text { (years) }\end{array}$ & Gender & $\begin{array}{l}\text { Major clinical } \\
\text { problem } \\
\text { and site of } \\
\text { infection } \\
\end{array}$ & $\begin{array}{l}\text { SOFA } \\
\text { Maximum }\end{array}$ & SAPS 2 & $\begin{array}{l}\text { Neurological } \\
\text { findings }\end{array}$ & $\begin{array}{l}\text { Lesion on } \\
\text { brain MRI } \\
\text { or CT }\end{array}$ & $\begin{array}{l}\text { EEG } \\
\text { background } \\
\text { activity }\end{array}$ & $\begin{array}{l}\text { Specific EEG } \\
\text { patterns }\end{array}$ & Prognosis \\
\hline 1 & 59 & $\mathrm{~F}$ & $\begin{array}{l}\text { Gastric surgery, } \\
\text { anastomotic } \\
\text { leak }\end{array}$ & 19 & 84 & $\begin{array}{l}\text { Clinical } \\
\text { seizure }\end{array}$ & No & Theta & $\begin{array}{l}\text { Generalized tonic- } \\
\text { clonic seizure record }\end{array}$ & Death \\
\hline 3 & 49 & $\mathrm{~F}$ & $\begin{array}{l}\text { Soft tissue } \\
\text { infection }\end{array}$ & 12 & 86 & $\begin{array}{l}\text { Clinical } \\
\text { seizure }\end{array}$ & No & Delta & $\begin{array}{l}\text { Electrophysiological } \\
\text { seizure }\end{array}$ & Death \\
\hline 5 & 73 & M & $\begin{array}{l}\text { Hepatocellular } \\
\text { carcinoma, } \\
\text { pneumonia }\end{array}$ & 16 & 68 & Coma & No & $\begin{array}{l}\text { Low } \\
\text { amplitude } \\
\text { non-reactive } \\
\text { rhythm }\end{array}$ & - & Death \\
\hline 6 & 22 & $\mathrm{~F}$ & $\begin{array}{l}\text { Acute } \\
\text { lymphoblastic } \\
\text { leukemia, } \\
\text { pneumonia }\end{array}$ & 9 & 53 & $\begin{array}{l}\text { Clinical } \\
\text { seizure }\end{array}$ & No & $\begin{array}{l}\text { Low } \\
\text { amplitude } \\
\text { non-reactive } \\
\text { rhythm }\end{array}$ & - & Death \\
\hline 7 & 61 & $\mathrm{~F}$ & $\begin{array}{l}\text { Gastric surgery, } \\
\text { anastomotic } \\
\text { leak }\end{array}$ & 9 & 49 & Coma & No & $\begin{array}{l}\text { Low } \\
\text { amplitude } \\
\text { non-reactive } \\
\text { rhythm }\end{array}$ & - & Survival \\
\hline 10 & 81 & $\mathrm{~F}$ & $\begin{array}{l}\text { Congestive } \\
\text { heart failure, } \\
\text { pneumonia }\end{array}$ & 7 & 51 & $\begin{array}{l}\text { Clinical } \\
\text { seizure }\end{array}$ & No & $\begin{array}{l}\text { Low } \\
\text { amplitude } \\
\text { non-reactive } \\
\text { rhythm }\end{array}$ & - & Death \\
\hline
\end{tabular}

SOFA: Sepsis-related Organ Failure Assessment score, SAPS: Simplified Acute Physiology score, MRI: Magnetic resonance imaging, CT: Computed tomography, EEG: Electroencephalography, F: Female, M: Male 


\begin{tabular}{|c|c|c|c|}
\hline Variables & Survivors $(\mathbf{n}=35)$ & Non-survivors $(n=26)$ & $\mathrm{p}$ \\
\hline \multicolumn{4}{|l|}{ During admission to ICU } \\
\hline Age (years) & $56(22-68)$ & $63(21-87)$ & 0.014 \\
\hline Gender F/M & 21/14 & 9/17 & 0.051 \\
\hline APACHE 2 score & $22(14-35)$ & $25(3-39)$ & 0.083 \\
\hline SOFA at admission & $9(3-15)$ & $12(5-24)$ & 0.017 \\
\hline SAPS 2 at admission & $45(15-90)$ & $58.5(16-78)$ & 0.220 \\
\hline \multicolumn{4}{|l|}{ Clinical findings during acute brain dysfunction } \\
\hline SOFA $(0-24)$ & $7(2-17)$ & $10.5(4-20)$ & 0.001 \\
\hline Renal SOFA (0-4) & $0(0-4)$ & $2.5(0-4)$ & 0.005 \\
\hline Hepatic SOFA (0-4) & $0(0-3)$ & $1(0-4)$ & 0.084 \\
\hline Delirium/Confusion & $16(46)$ & $4(15)$ & 0.013 \\
\hline Coma & $9(26)$ & $14(54)$ & 0.025 \\
\hline Presence of clinical seizures & $9(26)$ & $7(27)$ & 0.915 \\
\hline \multicolumn{4}{|l|}{ EEG findings during acute brain dysfunction } \\
\hline Background activity & & & 0.192 \\
\hline Theta & $14(40)$ & $10(39)$ & - \\
\hline Theta/delta & $16(46)$ & $9(35)$ & - \\
\hline Delta & $4(11)$ & $2(7)$ & - \\
\hline Absence of reactivation & $24(69)$ & $20(77)$ & 0.984 \\
\hline Low amplitude non-reactive background activity & $1(3)$ & $5(19)$ & 0.034 \\
\hline Low amplitude & $25(71)$ & $23(88)$ & 0.108 \\
\hline Generalized periodic epileptiform discharge & - & 1 & - \\
\hline Periodic lateralized epileptiform discharge & - & 1 & - \\
\hline Electrophysiological seizure & - & 2 & - \\
\hline Days in the intensive care unit & $21(3-103)$ & $16(4-72)$ & 0.470 \\
\hline Duration of hospitalization (days) & $44(17-120)$ & $36(6-80)$ & 0.026 \\
\hline
\end{tabular}

although studies conducted with EEG examinations with short durations in septic ICU patients were able to predict the clinical outcome $(11,26)$. Ideally, a continuous EEG should be performed in a separate room for a minimum of 24 hours. However, even though the precision in detecting the presence of intermittent electrophysiological seizures decreases in EEG examinations with short durations, EEG examination is still an important method to detect brain dysfunction, determine its severity, and to demonstrate the presence of non-convulsive status epilepticus in sepsis patients.

\section{CONCLUSION}

In conclusion, it should be noted that EEG monitoring can help determine the severity of sepsis-associated encephalopathy, in following up the encephalopathy, in determining whether it is accompanied by seizure or status epilepticus, and in prognosis.

\section{Acknowledgements}

The authors thank the personnel of the Multidisciplinary Critical Care Unit at the University of Istanbul for support and indebted to Mustafa Bahtiyar for assistance.

\section{Ethics}

Ethics Committee Approval: Ethics committee approval was obtained from the Ethics Committee of Istanbul University, İstanbul Faculty of Medicine (15.02.2012-2013/98).

Informed Consent: Written informed consent was obtained from the patients or their close relatives.

Peer-review: Externally peer-reviewed. 


\section{Authorship Contributions}

Concept: G.O., NB., F.E., Design: G.O., NB., F.E., Data Collection or Processing: G.O., N.B., P.E.Ö., A.A., F.E., Analysis or Interpretation: G.O., N.B., A.A., F.E., Literature Search: G.O., N.B., Writing: G.O., N.B.

Conflict of Interest: No conflict of interest was declared by the authors.

Financial Disclosure: This work was supported by Scientific Research Projects Coordination Unit of Istanbul University (Project number 35165)..

\section{REFERENCES}

1. Iacobone E, Bailly-Salin J, Polito A, Friedman D, Stevens RD, Sharshar T. Sepsis-associated encephalopathy and its differential diagnosis. Crit Care Med 2009;37(10 Suppl): 331-6.

2. Eidelman LA, Putterman D, Putterman C, Sprung CL. The spectrum of septic encephalopathy. Definitions, etiologies, and mortalities. JAMA 1996;275:470-3.

3. Gofton TE, Young GB. Sepsis-associated encephalopathy. Nat Rev Neurol 2012;8:557-66.

4. Ely EW, Shintani A, Truman B, Speroff T, Gordon SM, Harrell FE Jr, et al. Delirium as a predictor of mortality in mechanically ventilated patients in the intensive care unit. JAMA 2004;291:1753-62.

5. Iwashyna TJ, Ely EW, Smith DM, Langa KM. Long-term cognitive impairment and functional disability among survivors of severe sepsis. JAMA 2010;304:1787-94.

6. Sonneville R, Verdonk F, Rauturier C, Klein IF, Wolff M, Annane D, et al. Understanding brain dysfunction in sepsis. Ann Intensive Care 2013;3:15.

7. Young GB, Bolton CF, Archibald YM, Austin TW, Wells GA. The electroencephalogram in sepsis-associated encephalopathy. J Clin Neurophysiol 1992;9:145-52.

8. Kurtz P, Gaspard N, Wahl AS, Bauer RM, Hirsch LJ, Wunsch H, et al Continuous electroencephalography in a surgical intensive care unit. Intensive Care Med 2014;40:228-34

9. Oddo M, Carrera E, Claassen J, Mayer SA, Hirsch LJ. Continuous electroencephalography in the medical intensive care unit. Crit Care Med 2009;37:2051-6.

10. Hosokawa K, Gaspard N, Su F, Oddo M, Vincent JL, Taccone FS. Clinical neurophysiological assessment of sepsis-associated brain dysfunction: a systematic review. Crit Care 2014;18:674

11. Azabou E, Magalhaes E, Braconnier A, Yahiaoui L, Moneger G, Heming N, et al. Early Standard Electroencephalogram Abnormalities Predict Mortality in Septic Intensive Care Unit Patients. PLoS One 2015;10:e0139969.

12. Gilmore EJ, Gaspard N, Choi HA, Cohen E, Burkart KM, Chong DH, et al. Acute brain failure in severe sepsis: a prospective study in the medical intensive care unit utilizing continuous EEG monitoring. Intensive Care Med 2015;41:686-94.
13. Azabou E, Fischer C, Guerit JM, Annane D, Mauguiere F, Lofaso F, et al. Neurophysiological assessment of brain dysfunction in critically ill patients: an update. Neurol Sci 2017;38:715-26.

14. Dellinger RP, Levy MM, Rhodes A, Annane D, Gerlach H, Opal SM, et al. Surviving Sepsis Campaign: international guidelines for management of severe sepsis and septic shock, 2012. Intensive Care Med 2013;39:165228

15. Polito A, Eischwald F, Maho AL, Polito A, Azabou E, Annane D, et al Pattern of brain injury in the acute setting of human septic shock. Crit Care 2013;17:204.

16. Sessler CN, Gosnell MS, Grap MJ, Brophy GM, O’Neal PV, Keane KA, et al. The Richmond Agitation-Sedation Scale: validity and reliability in adult intensive care unit patients. Am J Respir Crit Care Med 2002;166:133844

17. Ely EW, Inouye SK, Bernard GR, Gordon S, Francis J, May L, et al. Delirium in mechanically ventilated patients: validity and reliability of the confusion assessment method for the intensive care unit (CAM-ICU). JAMA 2001;286:2703-10.

18. Posner JB, Saper CB, Schiff ND, Plum F. Examination of the comatose patient. In: Posner JB, Saper CB, Schiff ND, Plum F, editors. Plum and Posner's diagnosis of stupor and coma. 4th ed. Oxford: Oxford University Press; 2007. pp. 38-87.

19. Hirsch LJ, LaRoche SM, Gaspard N, Gerard E, Svoronos A, Herman ST, et al. American Clinical Neurophysiology Society's Standardized Critical Care EEG Terminology: 2012 version. J Clin Neurophysiol 2013;30:1-27.

20. Leitinger M, Beniczky S, Rohracher A, Gardella E, Kalss G, Qerama E, et al. Salzburg Consensus Criteria for Non-Convulsive Status Epilepticus-approach to clinical application. Epilepsy Behav 2015;49:158-63.

21. Oddo M, Taccone FS. How to monitor the brain in septic patients? Minerva Anestesiol 2015;81:776-88.

22. Claassen J, Taccone FS, Horn P, Holtkamp M, Stocchetti N, Oddo M, et al. Recommendations on the use of EEG monitoring in critically ill patients: consensus statement from the neurointensive care section of the ESICM. Intensive Care Med 2013;39:1337-51.

23. Guérit JM. Neurophysiological testing in neurocritical care. Curr Opin Crit Care 2010;16:98-104

24. Hughes CG, Patel MB, Pandharipande PP. Pathophysiology of acute brain dysfunction: what's the cause of all this confusion? Curr Opin Crit Care 2012;18:518-26

25. Schramm P, Klein KU, Falkenberg L, Berres M, Closhen D, Werhahn KJ et al. Impaired cerebrovascular autoregulation in patients with severe sepsis and sepsis-associated delirium. Crit Care 2012;16:181.

26. Sutter R, Stevens RD, Kaplan PW. Significance of triphasic waves in patients with acute encephalopathy: a nine-year cohort study. Clin Neurophysiol 2013;124:1952-8.

27. Andresen JM, Girard TD, Pandharipande PP, Davidson MA, Ely EW, Watson PL. Burst suppression on processed electroencephalography as a predictor of postcoma delirium in mechanically ventilated ICU patients. Crit Care Med 2014;42:2244-51.

28. Azabou E, Navarro V, Kubis N, Gavaret M, Heming N, Cariou A, et al Value and mechanisms of EEG reactivity in the prognosis of patients with impaired consciousness: a systematic review. Crit Care 2018;22:184. 